\title{
On Different Types of Heterogeneous Formal Contexts
}

\author{
Lubomír Antoni, Stanislav Krajči*, Ondrej Krídlo \\ Institute of Computer Science, Pavol Jozef Šafárik University in Košice, Slovakia
}

\begin{abstract}
We provide a generalization of Formal Concept Analysis that works with different types of the values in a heterogeneous formal context. An appropriate counterpart of the basic theorem on concept lattices is formulated. We suggest the transformation of the heterogeneous formal context to Galois connectional approach. Illustration on an example is included. Moreover, we show that this approach is a generalization of the multi-adjoint concept lattices proposed by Medina and Ojeda-Aciego. Finally, links between the proposed environment and related studies are stated.
\end{abstract}

Keywords: heterogeneous formal context, connectional approach, multi-adjointness, G-ideal

\section{Introduction}

Formal Concept Analysis is a method of data analysis, information management and knowledge representation. An input table data, represented as a formal context, describes relationship between a particular set of objects and a particular set of attributes. One of the main aims of Formal Concept Analysis is to construct formal concepts (interesting pairs of a particular set of objects and attributes) from a formal context. Classical formal context described in Ganter \& Wille's book [15] utilizes binary relation between a set of objects and a set of attributes. There are some other attempts that fuzzify the classical crisp context.

First, fuzzy subsets in both coordinates was done by Burusco \& Fuentes-Gonzales [12] and it was improved by Bělohlávek [3], [5] and Polland [30], [31]. Later, not so symmetric approaches were proposed by Ben Yahia \& Jaoua ([11]), Bělohlávek, Sklenář \& Zacpal ([8]) and Krajči ([17]) - it considers fuzzy sebsets only in the first coordinate and binary subsets in the second. All these environments were covered by generalized formal context [19], [20] that diversifies fuzziness of the subsets of attributes, fuzziness of the subsets of objects and moreover fuzziness of the table values.

Then Medina and Ojeda-Aciego brought the idea of multi-adjointness used in logic-programming [24],

\footnotetext{
*Corresponding author at: Institute of Computer Science, Faculty of Science, Pavol Jozef Šafárik University in Košice, Jesenná 5, 04154 Košice, Slovakia. E-mail adresses: lubomir.antoni@student.upjs.sk, stanislav.krajci@upjs.sk, ondrej.kridlo@upjs.sk.
}

[25], [26] to the Formal Concept Analysis too [21], [23]. This fact has inspired us to modify our generalized approach in such a way that it works with different lattice for every object, every attribute and every table field. This is the reason why we call this new approach heterogeneous. Another answer to the problem of data heterogeneity was given by Pócs in [28] and [29]. This approach also works with different lattices for every object and every attribute, but it gives a Galois connection to the table fields.

In this paper we describe different approaches with data heterogeneity and provide mutual relationships between diverse types of the formal contexts. Possible future work concludes the paper.

\section{Heterogeneous formal context}

First, we recall basic definitions and shortened results of heterogeneous approach from [2]. Further, interpretation of the heterogeneous formal concepts on an example is introduced.

Let $A$ and $B$ be non-empty sets. Let $\mathcal{P}=$ $\left(\left(P_{a, b}, \leq_{P_{a, b}}\right): a \in A, b \in B\right)$ be a system of posets and let $R$ be a function from $A \times B$ such that $R(a, b) \in P_{a, b}$ for all $a \in A$ and $b \in B$. Let $\mathcal{C}=$ $\left(\left(C_{a}, \leq_{C_{a}}\right): a \in A\right)$ and $\mathcal{D}=\left(\left(D_{b}, \leq_{D_{b}}\right): b \in B\right)$ be systems of complete lattices. (For simplicity, we omit the indices for all $\leq$ ?, since it is always clear which one is used.)

Let $\odot=\left(\left(\bullet_{a, b}\right): a \in A, b \in B\right)$ be a system of operations such that $\bullet_{a, b}$ is from $C_{a} \times D_{b}$ to $P_{a, b}$ and it is isotone and left-continuous in both arguments, i. e.

1a) $c_{1} \leq c_{2}$ implies $c_{1} \bullet_{a, b} d \leq c_{2} \bullet_{a, b} d$ for all $c_{1}, c_{2} \in$ $C_{a}$ and $d \in D_{b}$,

1b) $d_{1} \leq d_{2}$ implies $c \bullet_{a, b} d_{1} \leq c \bullet_{a, b} d_{2}$ for all $c \in C_{a}$ and $d_{1}, d_{2} \in D_{b}$,

2a) if $c \bullet_{a, b} d \leq p$ for some $d \in D_{b}, p \in P_{a, b}$ and for all $c \in X \subseteq C_{a}$ then $\sup X \bullet_{a, b} d \leq p$,

2b) if $c \bullet_{a, b} d \leq p$ for some $c \in C_{a}, p \in P_{a, b}$ and for all $d \in Y \subseteq D_{b}$ then $c \bullet_{a, b}$ sup $Y \leq p$.

Then we call the tuple $\langle A, B, \mathcal{P}, R, \mathcal{C}, \mathcal{D}, \odot\rangle$ a heterogeneous formal context.

Let $F$ be a set of all functions $f$ with a domain $A$ such that $f(a) \in C_{a}$ for all $a \in A$ (more formally, $F=\prod_{a \in A} C_{a}$ ). Let $G$ be a set of all functions $g$ with a domain $B$ such that $g(b) \in D_{b}$ for all $b \in B$. (i. e., $G=\prod_{b \in B} D_{b}$ ). 
We define the mapping $\nearrow: G \rightarrow F$. If $g \in G$, then $\nearrow(g) \in F$ is defined by

$$
\begin{aligned}
(\nearrow(g))(a)= & \sup \left\{c \in C_{a}:\right. \\
& \left.(\forall b \in B) c \bullet_{a, b} g(b) \leq R(a, b)\right\} .
\end{aligned}
$$

Symmetrically, we define the mapping $\swarrow: F \rightarrow$ $G$. If $f \in F$, then $\swarrow(f) \in G$ is defined as

$$
\begin{aligned}
(\swarrow(f))(b)= & \sup \left\{d \in D_{b}:\right. \\
& \left.(\forall a \in A) f(a) \bullet_{a, b} d \leq R(a, b)\right\} .
\end{aligned}
$$

The mappings $\nearrow$ and $\swarrow$ defined in this way have worthwile properties.

Theorem 1 Let $f \in F$ and $g \in G$. Then the following conditions are equivalent:

1) $f \leq \nearrow(g)$.

2) $g \leq \swarrow(f)$.

3) $f(a) \bullet_{a, b} g(b) \leq R(a, b)$ for all $a \in A$ and $b \in B$.

Corrolary 1 Mappings $\nearrow$ and $\swarrow$ form a Galois connection.

In what follows, we use a Galois connection $(\nearrow, \swarrow)$ for the concept lattice construction via classical Ganter-Wille's approach from [15].

Lemma 1 1) Let $\left\{g_{i}: i \in I\right\} \subseteq G$. Then

$$
\nearrow\left(\bigvee_{i \in I} g_{i}\right)=\bigwedge_{i \in I} \nearrow\left(g_{i}\right)
$$

2) Let $\left\{f_{i}: i \in I\right\} \subseteq F$. Then

$$
\swarrow\left(\bigvee_{i \in I} f_{i}\right)=\bigwedge_{i \in I} \swarrow\left(f_{i}\right) .
$$

We call a pair $\langle g, f\rangle$ from $G \times F$ such that $\nearrow(g)=$ $f$ and $\swarrow(f)=g$ a heterogeneous formal concept.

Lemma 2 If $\left\langle g_{1}, f_{1}\right\rangle$ and $\left\langle g_{2}, f_{2}\right\rangle$ are concepts, then $g_{1} \leq g_{2}$ iff $f_{1} \geq f_{2}$.

This lemma allows us to define the following ordering of concepts: $\left\langle g_{1}, f_{1}\right\rangle \leq\left\langle g_{2}, f_{2}\right\rangle$ iff $g_{1} \leq g_{2}$ (or equivalently $f_{1} \geq f_{2}$ ).

We call the poset of all concepts ordered by $\leq$ a heterogeneous concept lattice, denoted by $\operatorname{HCL}(A, B, \mathcal{P}, R, \mathcal{C}, \mathcal{D}, \odot, \swarrow, \nearrow, \leq)$.

The following theorem shows that this is in reality a lattice.

Theorem 2 (The Basic Theorem on Heterogeneous Concept Lattices)

1) $A$ heterogeneous concept lattice $\operatorname{HCL}(A, B, \mathcal{P}, R, \mathcal{C}, \mathcal{D}, \odot, \swarrow, \nearrow, \leq)$ is a complete lattice in which

$$
\bigwedge_{i \in I}\left\langle g_{i}, f_{i}\right\rangle=\left\langle\bigwedge_{i \in I} g_{i}, \nearrow\left(\swarrow\left(\bigvee_{i \in I} f_{i}\right)\right)\right\rangle
$$

and

$$
\bigvee_{i \in I}\left\langle g_{i}, f_{i}\right\rangle=\left\langle\swarrow\left(\nearrow\left(\bigvee_{i \in I} g_{i}\right)\right), \bigwedge_{i \in I} f_{i}\right\rangle .
$$

2) For each $a \in A$ and $b \in B$, let $P_{a, b}$ have the least element $0_{P_{a, b}}$ such that $0_{C_{a}} \bullet_{a, b} d=$ $c \bullet_{a, b} 0_{D_{b}}=0_{P_{a, b}}$ for all $c \in C_{a}, d \in D_{b}$. Then a complete lattice $L$ is isomorphic to $\operatorname{HCL}(A, B, \mathcal{P}, R, \mathcal{C}, \mathcal{D}, \odot, \swarrow, \nearrow, \leq)$ if and only if there are mappings $\alpha: \bigcup_{a \in A}\left(\{a\} \times C_{a}\right) \rightarrow L$ and $\beta: \bigcup_{b \in B}\left(\{b\} \times D_{b}\right) \rightarrow L$ such that:

a) $\alpha$ does not increase in the second argument (for a fixed first argument).

b) $\beta$ does not decrease in the second argument (for a fixed first argument).

c) $\operatorname{Rng}(\alpha)$ is inf-dense in $L$.

d) $\operatorname{Rng}(\beta)$ is sup-dense in $L$.

e) For $a \in A, b \in B$ and $c \in C_{a}, d \in D_{b}$

\begin{tabular}{|c|c|c|c|c|}
\hline \multirow{2}{*}{\multicolumn{2}{|c|}{ objects }} & water & services & lake \\
\hline & & - ${ }_{\text {hot }}^{\text {cold }}$ & $\mathrm{in}+\mathrm{tv}$ & † yes \\
\hline 恿 & $\prod_{\emptyset}^{\mathrm{Sa}+\mathrm{Su}} 1 / 2$ & $\iota_{0}^{1}$ & $\cdot \begin{array}{l}1 \\
1 / 2 \\
0\end{array}$ & $\iota_{0}^{1}$ \\
\hline$\stackrel{8}{\circ}$ & $\mathrm{Sa}+\mathrm{Su}$ & $\left\{\begin{array}{l}1 \\
1 / 2 \\
0\end{array}\right.$ & 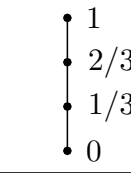 & $\cdot \begin{array}{l}1 \\
1 / 2 \\
0\end{array}$ \\
\hline 胥 & $\prod_{\emptyset}^{\mathrm{Sa}+\mathrm{Su}} 1 / 2$ & $\left\lceil_{0}^{1}\right.$ & 0 & $\mathfrak{\bullet}^{1} 1 / 2$ \\
\hline 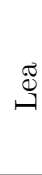 & $\varliminf_{\emptyset}^{\mathrm{Sa}+\mathrm{Su}} 1 / 2$ & $\left\lceil_{0}^{1}\right.$ & $\cdot \begin{array}{l}1 \\
1 / 2 \\
0\end{array}$ & 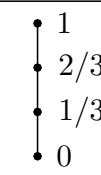 \\
\hline$\stackrel{8}{5}$ & $\varliminf_{\emptyset}^{\mathrm{Sa}+\mathrm{Su}} 1 / 2$ & $\left\{\begin{array}{l}1 \\
1 / 2 \\
0\end{array}\right.$ & 0 & • 1 \\
\hline 四 & $\mathrm{Sa}+\mathrm{Su}$ & $\complement_{0}^{1}$ & 0 & $\begin{array}{l}\mathfrak{l}^{1} \\
2 / 3 \\
1 / 3 \\
0\end{array}$ \\
\hline
\end{tabular}

$$
\alpha(a, c) \geq \beta(b, d)
$$

if and only if

$$
c \bullet_{a, b} d \leq R(a, b) .
$$

For self-contained proof see [2].

The following figure is a good candidate to illustrate the underlying structures of heterogeneous formal context.

Figure 1: List of possible values for objects and attributes 
The set of objects $(B)$ in Figure 1 corresponds to people who are going to stay at a cottage together. The complete lattices for objects $\left(D_{b}, b \in B\right)$ express different length of staying (no stay, one arbitrary day, only Saturday, only Sunday, both days). For instance, Eva has three preferences for staying: not at all, one day (it does not matter if Saturday or Sunday) or both days $\left(D_{\text {Eva }}\right)$. But Joe has four preferences: not at all, only Saturday, only Sunday (he distinguishes if Saturday or Sunday) or both days $\left(D_{\text {Joe }}\right)$.

The set of attributes $(A)$ responds to the type of cottage conditions. The complete lattices for attributes $\left(C_{a}, a \in A\right)$ express different degrees of some specific cottage condition. The water conditions contain two degrees: hor or cold $\left(C_{\text {water }}\right)$. There are four possibilities for services: internet and television, internet only, television only, or nothing at all $\left(C_{\text {services }}\right)$.

The degrees of each table value $\left(P_{a, b}, a \in A, b \in\right.$ $B)$ refer to the degrees of discomfort that a particular person admits at a particular cottage condition (no discomfort, one-third discomfort, partial discomfort, two-thirds discomfort, large discomfort, discomfort on length of stay, discomfort on services).

Each person can accept different degrees of discomfort for longterm preferences $\left(\bullet_{a, b}, a \in A, b \in\right.$ $B)$. For instance, $\bullet_{\text {services, Eva }}$ is from $C_{\text {services }} \times D_{\text {Eva }}$ to $P_{\text {services,Eva, }}$, where $P_{\text {services,Eva }}=\{0,1 / 2,1\}$ denotes comfort, partial discomfort and large discomfort, respectively, for Eva. Higher value from $P_{a, b}$ corresponds to higher discomfort, i. e. personal satisfaction is less with higher degree. That is in opposite with natural expectation, but this follows from assumptions of our heterogeneous approach. Moreover, notice that the structures from Figure 1 in the first and fourth row equal. This is only a special case, because our approach allows us to use a different lattice for different object, a different lattice for different attribute and also a different poset for different table field. In what follows, we consider longterm preferences (Figure 2 - Figure 5) just for two people and two conditions from Figure 1.

\begin{tabular}{|c|cccc|}
\hline$\bullet$ services,Eva & in+tv & in & tv & no \\
\hline$\emptyset$ & 0 & 0 & 0 & 0 \\
$1 / 2$ & 0 & $1 / 2$ & 1 & 1 \\
$\mathrm{Sa}+\mathrm{Su}$ & 0 & 1 & 1 & 1 \\
\hline
\end{tabular}

Figure 2: Longterm preferences for Eva and services

Another point are shortterm preferences that can be expressed by function $R$ (Figure 6 ). It represents some actual circumstances or some actual willingness to deal with discomfort (from short-term point of view). For instance, if $R$ (services, Eva) $=1 / 2$, it means that Eva will accept neither all services nor a maximum of one arbitrary day with internet only, because these cases for Eva are less than or equal to $1 / 2$ in Figure 2.

\begin{tabular}{|c|cc|}
\hline • water,Eva & hot & cold \\
\hline$\emptyset$ & 0 & 0 \\
$1 / 2$ & 0 & 1 \\
$\mathrm{Sa}+\mathrm{Su}$ & 0 & 1 \\
\hline
\end{tabular}

Figure 3: Longterm preferences for Eva and water

\begin{tabular}{|c|cc|}
\hline - water,Joe & hot & cold \\
\hline$\emptyset$ & 0 & 0 \\
$\mathrm{Sa}$ & 0 & $1 / 2$ \\
$\mathrm{Su}$ & 0 & 1 \\
$\mathrm{Sa}+\mathrm{Su}$ & 0 & 1 \\
\hline
\end{tabular}

Figure 4: Longterm preferences for Joe and water

\begin{tabular}{|c|cccc|}
\hline • services,Joe & in+tv & in & tv & no \\
\hline$\emptyset$ & 0 & 0 & 0 & 0 \\
$\mathrm{Sa}$ & 0 & $1 / 3$ & $2 / 3$ & $2 / 3$ \\
$\mathrm{Su}$ & 0 & $1 / 3$ & 1 & 1 \\
$\mathrm{Sa}+\mathrm{Su}$ & 0 & $1 / 3$ & 1 & 1 \\
\hline
\end{tabular}

Figure 5: Longterm preferences for Joe and services

\begin{tabular}{|c||c|c|}
\hline & water & services \\
\hline \hline Eva & 1 & $1 / 2$ \\
\hline Joe & $1 / 2$ & $2 / 3$ \\
\hline
\end{tabular}

Figure 6: Shortterm preferences

We use mappings $(\nearrow, \swarrow)$ to identify the required cottage conditions as follows. Mapping $(\swarrow(f))(b)$ indicates maximization of the number of days spent at the cottage for specific water and services conditions that return the greatest degree of discomfort accepted by a person. For instance, for $f$ (water) $=$ hot, $f$ (services) $=$ in we obtain $(\swarrow(f))($ Eva $)=1 / 2$, which means that hot water and internet only correspond to a maximum stay of 1 day for Eva. Mapping $(\nearrow(g))(a)$ indicates the worst water or services conditions at the cottage for a specific number of days that return the greatest degree of discomfort accepted. For instance, if $g($ Eva $)=1 / 2, g($ Joe $)=\mathrm{Sa}$, then we obtain $(\nearrow(g))($ water $)=$ cold, which means that Eva's stay for 1 day and Joe's stay on Saturday correspond to the possibility of cold water at the cottage. In another example, if $g(\mathrm{Eva})=\mathrm{Sa}+\mathrm{Su}, g(\mathrm{Joe})=\mathrm{Sa}$, then we obtain $(\nearrow(g))$ (services) $=$ in + tv, whereby a cottage with an internet connection and $\mathrm{TV}$ is the 
worst possible case if Eva stays on Saturday and Sunday and Joe stays on Saturday.

Having expressed all personal preferences (longterm and shortterm), all heterogeneous formal concepts by the heterogeneous concept lattice construction are generated. Every concept has natural intepretation. It stated the worst case of cottage conditions to stay specific number of days. The list of formal concepts for two people and two conditions is shown in Figure 7.

\begin{tabular}{|cc|cc|}
\hline \multicolumn{2}{|c|}{ extents } & \multicolumn{2}{c|}{ intents } \\
Eva & Joe & water & services \\
\hline \hline$\emptyset$ & $\mathrm{Sa}$ & cold & no \\
\hline $1 / 2$ & $\mathrm{Sa}$ & cold & in \\
\hline $1 / 2$ & $\mathrm{Sa}+\mathrm{Su}$ & hot & in \\
\hline $\mathrm{Sa}+\mathrm{Su}$ & $\mathrm{Sa}$ & cold & in $+\mathrm{tv}$ \\
\hline $\mathrm{Sa}+\mathrm{Su}$ & $\mathrm{Sa}+\mathrm{Su}$ & hot & in $+\mathrm{tv}$ \\
\hline
\end{tabular}

Figure 7: List of heterogeneous formal concepts for two people and two conditions

For instance, last concept indicates full stay of both people only at the cottage with hot water, internet connection and television. In contrary, second concept states that in case of cold water and internet connection only, the number of days at the cottage will be maximal one arbitrary day for Eva and only Saturday for Joe. Note that intents do not include the possibility of hot water and no services simultaneously. In this case we obtain $\swarrow$ (hot, no $)=(\emptyset$, Sa $)$ and subsequently $\nearrow(\emptyset, \mathrm{Sa})=($ cold, no $)$. This can be interpreted as superfluous conditions for Joe's stay on Saturday and maybe a cheaper cottage can be chosen.

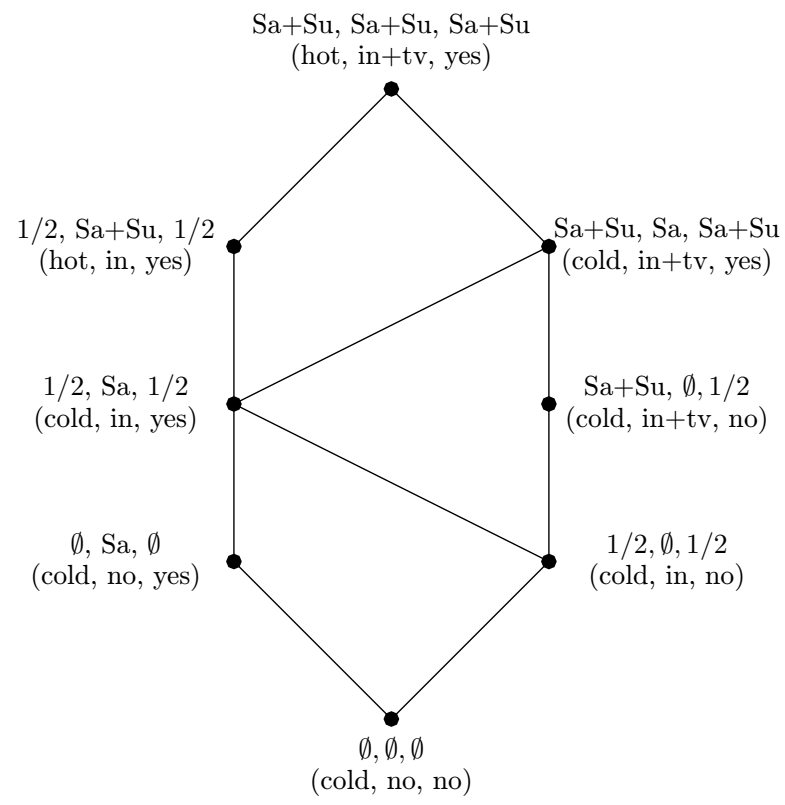

Figure 8: Heterogeneous concept lattice for three people and three conditions
Another possibility is to make computation of the heterogeneous formal concepts for three people and three cottage conditions. The resulting heterogeneous concept lattice with eight ordered concepts is illustrated in Figure 8. The first row of every concept refers to extent, the second row expresses its intent. And likewise, full stay $(\mathrm{Sa}+\mathrm{Su})$ for three people is associated only with a cottage having hot water, internet connection, television and, in addition, lake available.

There is also possible to consider a similar example of heterogeneous formal context based on job preferences whereby table values express dissatisfaction with type of contract and job conditions like salary, language requirements. Likewise, a higher value correspond to a higher dissatisfaction that is in opposite with a natural expectation, but it comes from assumptions of our approach.

\section{Galois connectional formal context}

The main aim of this section is to recall the shortened definitions and results of approach from [28], [29] which is inspired by the (homogeneous) approach from [32].

Let $A$ and $B$ be non-empty sets. Let $\mathcal{C}=$ $\left(\left(C_{a}, \leq_{C_{a}}\right): a \in A\right)$ and $\mathcal{D}=\left(\left(D_{b}, \leq_{D_{b}}\right): b \in B\right)$ be systems of complete lattices. Let $\mathcal{G}=\left(\left(\phi_{a, b}, \psi_{a, b}\right)\right.$ : $a \in A, b \in B)$ be a system of (antitone) Galois connection s.t. $\left(\phi_{a, b}, \psi_{a, b}\right)$ is a Galois connection from $\left(C_{a}, \leq_{C_{a}}\right)$ to $\left(D_{b}, \leq_{D_{b}}\right)$. (Again we omit the indices of all noticed $\leq_{\text {?.) }}$

Define the following mapping $\uparrow: G \rightarrow F$ : If $g \in G$ then $\uparrow(g) \in F$ is defined by

$$
(\uparrow(g))(a)=\bigwedge_{b \in B} \psi_{a, b}(g(b)) .
$$

Symmetrically define the mapping $\downarrow: F \rightarrow G$ : If $f \in F$ then $\downarrow(f) \in G$ is defined as following:

$$
(\downarrow(f))(b)=\bigwedge_{a \in A} \phi_{a, b}(f(a)) .
$$

Theorem $3(\uparrow, \downarrow)$ is a Galois connection.

Hence the classical Ganter-Wille's process can be used for the concept lattice construction, so it can be obtained the following.

A concept in this approach is a pair $\langle g, f\rangle$ from $G \times F$ such that $\uparrow(g)=f$ and $\downarrow(f)=g$.

Lemma 3 If $\left\langle g_{1}, f_{1}\right\rangle$ and $\left\langle g_{2}, f_{2}\right\rangle$ are concepts then $g_{1} \leq g_{2}$ iff $f_{1} \geq f_{2}$.

This lemma allows to define the following ordering of concepts: $\left\langle g_{1}, f_{1}\right\rangle \leq\left\langle g_{2}, f_{2}\right\rangle$ iff $g_{1} \leq g_{2}$ (or equivalently $f_{1} \geq f_{2}$ ).

The poset of all such concepts ordered by $\leq$ will be called a connectional concept lattice and denoted by $\operatorname{CCL}(A, B, \mathcal{C}, \mathcal{D}, \mathcal{G}, \downarrow, \uparrow, \leq)$. 
Theorem 4 (The Basic Theorem on Connectional Concept Lattices)

1) A connectional concept lattice $\operatorname{CCL}(A, B, \mathcal{C}, \mathcal{D}, \mathcal{G}, \downarrow, \uparrow, \leq) \quad$ is $\quad$ a complete lattice in which

$$
\bigwedge_{i \in I}\left\langle g_{i}, f_{i}\right\rangle=\left\langle\bigwedge_{i \in I} g_{i} \uparrow\left(\downarrow\left(\bigvee_{i \in I} f_{i}\right)\right)\right\rangle
$$

and

$$
\bigvee_{i \in I}\left\langle g_{i}, f_{i}\right\rangle=\left\langle\downarrow\left(\uparrow\left(\bigvee_{i \in I} g_{i}\right)\right), \bigwedge_{i \in I} f_{i}\right\rangle .
$$

2) A complete lattice $L$ is isomorphic to $\operatorname{CCL}(A, B, \mathcal{C}, \mathcal{D}, \mathcal{G}, \downarrow, \uparrow, \leq)$ if and only if there are mappings $\alpha: \bigcup_{a \in A}\left(\{a\} \times C_{a}\right) \rightarrow L$ and $\beta: \bigcup_{b \in B}\left(\{b\} \times D_{b}\right) \rightarrow L$ such that for every $a \in A, b \in B$ and $c \in C_{a}, d \in D_{b}$

$$
\begin{gathered}
\alpha(a, c) \geq \beta(b, d) \quad \text { iff } \quad d \leq \phi_{a, b}(c) \\
\text { iff } \quad c \leq \psi_{a, b}(d) .
\end{gathered}
$$

\section{From heterogeneous to connectional context}

The notion of G-ideal defined in [33] is useful for transformation from heterogeneous context to connectional one.

Let $\left(L, \leq_{L}\right),\left(M, \leq_{M}\right)$ be complete lattices. Then $J \subseteq L \times M$ is called a $G$-ideal of $L \times M$ when the following conditions hold:

1) If $(\ell, m) \in J$ and $\left(\ell^{\prime}, m^{\prime}\right) \leq(\ell, m)$ (coordinatewise, i.e. $\ell^{\prime} \leq \ell$ and $\left.m^{\prime} \leq m\right)$ then $\left(\ell^{\prime}, m^{\prime}\right) \in J$.

2) If $\left\{\left(\ell_{i}, m_{i}\right): i \in I\right\} \subseteq J$ then

$\left(\bigvee_{i \in I} \ell_{i}, \bigwedge_{i \in I} m_{i}\right),\left(\bigwedge_{i \in I} \ell_{i}, \bigvee_{i \in I} m_{i}\right) \in J$.

If $I=\emptyset$ then $\left(0_{L}, 1_{M}\right),\left(1_{L}, 0_{M}\right) \in J$.

The following theorem shows correspondences between Galois connections and G-ideals.

Theorem 5 [33] Let $\left(L, \leq_{L}\right),\left(M, \leq_{M}\right)$ be complete lattices.

1) If $(\phi, \psi)$ is an (antitone) Galois connection from $\left(L, \leq_{L}\right)$ to $\left(M, \leq_{M}\right)$ then

$$
\left\{(\ell, m): \phi(\ell) \geq_{M} m\right\}=\left\{(\ell, m): \psi(m) \geq_{L} \ell\right\}
$$

is a $G$-ideal on $L \times M$.

2) If $J$ is a $G$-ideal on $L \times M$ then the mappings $\phi: L \rightarrow M$ and $\psi: M \rightarrow L$ defined by

$$
\phi(\ell)=\bigvee\{m \in M:(\ell, m) \in J\}
$$

and

$$
\psi(m)=\bigvee\{\ell \in L:(\ell, m) \in J\}
$$

form a Galois connection from $\left(L, \leq_{L}\right)$ to $\left(M, \leq_{M}\right)$.
Moreover, this correspondences between Galois connections and $G$-ideals are each other inverse.

The previous theorem is used in the following way:

Lemma 4 [28] Let $\left(L, \leq_{L}\right),\left(M, \leq_{M}\right)$ be complete lattices, $\left(P, \leq_{P}\right)$ be poset and $\bullet: L \times M \rightarrow P$ is isotone and left-continuous in both arguments. Then

$$
\{(\ell, m): \ell \bullet m \leq p\}
$$

is a G-ideal.

And now assume that we have a heterogeneous concept lattice $\operatorname{HCL}(A, B, \mathcal{P}, R, \mathcal{C}, \mathcal{D}, \odot, \swarrow, \nearrow, \leq)$. For each $a \in A$ and $b \in B$ define

$$
J_{a, b}=\left\{(c, d) \in C_{a} \times D_{b}: c \bullet_{a, b} d \leq R(a, b)\right\},
$$

by the previous lemma we know that $J_{a, b}$ is a Gideal on $C_{a} \times D_{b}$. Then again for each $a \in A$ and $b \in B$ define the mappings $\phi_{a, b}: C_{a} \rightarrow D_{b}$ and $\psi_{a, b}: D_{b} \rightarrow C_{a}$ expressed by

$$
\phi_{a, b}(c)=\bigvee\left\{d \in D_{b}:(c, d) \in J_{a, b}\right\}
$$

and

$$
\psi_{a, b}(d)=\bigvee\left\{c \in C_{a}:(c, d) \in J_{a, b}\right\}
$$

and we know by Theorem 5 that $\left(\phi_{a, b}, \psi_{a, b}\right)$ is a Galois connection from $C_{a}$ to $D_{b}$. Finally, we define mappings $\downarrow$ and $\uparrow$ as before:

$$
(\uparrow(g))(a)=\bigwedge_{b \in B} \psi_{a, b}(g(b))
$$

and

$$
(\downarrow(f))(b)=\bigwedge_{a \in A} \phi_{a, b}(f(a)) .
$$

Finally, one can obtained that the corresponding mappings for heterogeneous and Galois connectional concept lattice construction equal by previous formulation.

Theorem $6(\uparrow, \downarrow)=(\nearrow, \swarrow)$.

For the proof see [1].

\section{From connectional to heterogeneous context}

In this section we show opposite direction, namely that the heterogeneous approach covers the connectional one. The transformation of connectional approach to heterogenenous uses the following way:

Firstly, one fact from [33] analogous to Lemma 1:

Lemma 5 Let $\left(L, \leq_{L}\right),\left(M, \leq_{M}\right)$ be complete lattices and $(\phi, \psi)$ be a Galois connection from $\left(L, \leq_{L}\right)$ to $\left(M, \leq_{M}\right)$. 
1) For arbitrary subset $\left\{\ell_{i}: i \in I\right\}$ of $L$

$$
\phi\left(\bigvee_{i \in I} \ell_{i}\right)=\bigwedge_{i \in I} \phi\left(\ell_{i}\right)
$$

2) For arbitrary subset $\left\{m_{i}: i \in I\right\}$ of $M$

$$
\psi\left(\bigvee_{i \in I} m_{i}\right)=\bigwedge_{i \in I} \psi\left(m_{i}\right)
$$

We use it for the proof that specially defined operation • fulfills all necessary assumptions of our heterogeneous environment.

Theorem 7 Let $\left(L, \leq_{L}\right),\left(M, \leq_{M}\right)$ be complete lattices and $(\phi, \psi)$ be a Galois connection from $\left(L, \leq_{L}\right)$ to $\left(M, \leq_{M}\right)$. Let $\bullet: L \times M \rightarrow(\{0,1\}, \leq)$ be defined in the following way:

$$
\ell \bullet m= \begin{cases}0 & \text { if } \phi(\ell) \geq m(\text { iff } \psi(m) \geq \ell) \\ 1 & \text { elsewhere }\end{cases}
$$

Then • is isotone and left-continuous in both arguments.

And now assume that we have a connectional concept lattice $\operatorname{CCL}(A, B, \mathcal{C}, \mathcal{D}, \mathcal{G}, \downarrow, \uparrow, \leq)$. For each $a \in A$ and $b \in B$ take the same $P_{a, b}=(\{0,1\}, \leq)$, $R(a, b)=0$ (sic!) and $\bullet_{a, b}: C_{a} \times D_{b} \rightarrow P_{a, b}$ such that for all $c \in C_{a}$ and $d \in D_{b}$,

$$
c \bullet_{a, b} d= \begin{cases}0 & \text { if } \phi_{a, b}(c) \geq d\left(\text { iff } \psi_{a, b}(d) \geq c\right), \\ 1 & \text { elsewhere }\end{cases}
$$

By Theorem $7 \bullet_{a, b}$ is isotone and left-continuous in both arguments, so we have a frame for heterogeneous approach and we can define the mappings $\nearrow$ and $\swarrow$ as before.

Theorem $8(\nearrow, \swarrow)=(\uparrow, \downarrow)$.

For the proof see [1].

So the previous formulation is the answer for transformation. Likewise, from Galois connections we can construct system of operations $\odot=\left(\left(\bullet \bullet_{a, b}\right)\right.$ : $a \in A, b \in B$ ) defined in Section 2 .

\section{From heterogeneous to multi-adjoint context}

Multi-adjoint formal context in [21] introduced by Medina and Ojeda-Aciego works with adjoints to -'s in a non-commutative environment. Recall this notion:

Let $\left(C, \leq_{C}\right),\left(D, \leq_{D}\right)$ and $\left(P, \leq_{P}\right)$ be posets. The triple $\left(\bullet, \rightarrow_{1}, \rightarrow_{2}\right)$ is called adjoint triple or implication triple if $\bullet:(C \times D) \rightarrow P, \rightarrow_{1}:(D \times P) \rightarrow C$, $\rightarrow_{2}:(C \times P) \rightarrow D$ and

$$
(c \bullet d) \leq p \text { iff } c \leq\left(d \rightarrow_{1} p\right) \text { iff } d \leq\left(c \rightarrow_{2} p\right) .
$$

(In the case $C=D$ and the commutative $\bullet$ both arrows are identical.)

Moreover, in case that $\left(C, \leq_{C}\right),\left(D, \leq_{D}\right)$ are complete lattices and $\left(P, \leq_{P}\right)$ is a poset, then a multi-adjoint frame is a tuple denoted as $\left(C, D, P, \bullet_{b}\right.$ for $\left.b \in B\right)$, where for all $b \in B$ is $\left(\bullet_{b}, \rightarrow_{1_{b}}, \rightarrow_{2_{b}}\right)$ an adjoint triple with respect to $C, D, P$.

Finally, define a multi-adjoint context as a tuple $(A, B, R, \sigma)$ such that $A$ and $B$ are set of attributes and set of objects, respectively, $R$ is a function from $A \times B$ such that $R(a, b) \in P$, for all $a \in A$ and $b \in B$ and $\sigma$ is a mapping that associates any object from $B$ with some particular adjoint triple in the multiadjoint frame.

This multi-adjointness approach has not very aesthetic property: it takes only $\bullet_{b}$ for $b \in B$ without any reference to $A$. In our heterogeneous approch, we symmetrize it and consider operations $\bullet_{a, b}$ for each pair $(a, b) \in A \times B$. This, of course, diversifies and generalizes [21]. Moreover, we need not the equal lattices for all $b \in B$ and/or all $a \in A$.

Medina, Ojeda-Aciego and Ruiz-Calviño in [23] consider situation that we have written a scientific paper and have to decide which journal to choose for submitting. Set of objects consists of particular scientific journal (AMC, CAMWA, FSS, IJUFKS, JIFS, ...) and set of attributes includes journal properties as impact factor, immediacy index, cited half-life and best position. Furthermore, problem consists in finding a multi-adjoint concept which represent the suitable journal to submit. They assign a different adjoint triple to the journals listed under a different category. For instance, an operations $\bullet$ IJUFKS $=\bullet$ JIFS, because IJUFKS and JIFS are the journals listed under the Artificial Intelligence category. Nevertheless, $\bullet$ JIFS and $\bullet$ AMC is different, because AMC is listed under different category than JIFC. The same adjoint triple is assigned for instance for $\bullet$ AMC $=\bullet$ CAMWA .

Having looked at our cottage example, situation that the same adjoint triple is assigned to two journals corresponds to the same longterm preferences for two people. This means, for instance, that $\bullet$ water,Eva $=\bullet \bullet_{\text {water,Lea }}, \bullet_{\text {services,Eva }}=$

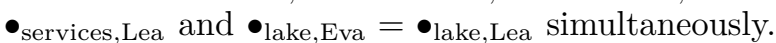
Another important difference is that all attributes are evaluated in the same complete lattice $\left(C_{\text {water }}=\right.$ $\left.C_{\text {services }}=C_{\text {lake }}\right)$, all objects have the same complete lattice $\left(D_{\text {Eva }}=D_{\text {Joe }}=D_{\text {Lea }}=\ldots\right)$ and moreover every table field takes the values from the same poset $\left(P_{\text {water,Eva }}=P_{\text {services }, \text { Joe }}=P_{\text {lake,Lea }}=\ldots\right)$.

On the other hand, for each operation $\bullet$ which is isotone and left-continuous in both arguments, there are operations $\rightarrow_{1}$ and $\rightarrow_{2}$ s.t. $\left(\bullet, \rightarrow_{1}, \rightarrow_{2}\right)$ is an adjoint triple - it is enough to define

$$
d \rightarrow_{1} p=\sup \left\{c \in C: c \bullet d \leq_{P} p\right\}
$$

and symmetrically

$$
c \rightarrow_{2} p=\sup \left\{d \in D: c \bullet d \leq_{P} p\right\} .
$$


Obviously, $c \bullet d \leq_{P} p$ means $c \in\left\{c^{\prime} \in C: c^{\prime} \bullet d \leq_{P}\right.$ $p\}$ hence $c \leq_{C} \sup \left\{c^{\prime} \in C: c^{\prime} \bullet d \leq_{P} p\right\}=d \rightarrow_{1} p$.

Conversely, by the left-continuity of $\bullet$ in the first argument we have $\sup \left\{c^{\prime} \in C: c^{\prime} \bullet d \leq_{P} p\right\} \bullet d \leq_{P} p$, i. e. $\left(d \rightarrow_{1} p\right) \bullet d \leq_{P} p$. So, if $c \leq_{C} d \rightarrow_{1} p$ then by the isotonity of $\bullet$ in the first argument we have $c \bullet d \leq_{P} p$.

The dual properties of $\rightarrow_{2}$ can be proved symmetrically. This means that it is equivalent to work with adjoint triples and to work with isotone and left-continuous functions.

\section{Conclusion}

In this paper we introduce different types of the formal contexts with data heterogeneity - heterogeneous, Galois connectional and multi-adjointness environment. The main idea of our heterogenenous approach is to diversify all that can be diversified and it is interesting that the process of concept lattice construction still works. Hence, intuitively, it allows to use the Formal Concept Analysis also for tables with data of different types.

The comparison of our heterogeneous environment with connectional approach is in the following table.

\begin{tabular}{|c|c|}
\hline $\begin{array}{l}\text { heterogeneous } \\
\text { approach }\end{array}$ & $\begin{array}{l}\text { connectional } \\
\text { approach }\end{array}$ \\
\hline $\begin{array}{l}\text { longterm and } \\
\text { shortterm preferences }\end{array}$ & $\begin{array}{l}\text { all information in } \\
\text { Galois connections }\end{array}$ \\
\hline $\begin{array}{l}\text { metadata and } \\
\text { data divided }\end{array}$ & $\begin{array}{l}\text { metadata and } \\
\text { data mixed }\end{array}$ \\
\hline $\begin{array}{l}\text { easier to } \\
\text { illustrate on example }\end{array}$ & $\begin{array}{l}\text { difficult to } \\
\text { interpret }\end{array}$ \\
\hline $\begin{array}{l}\text { can be expressed } \\
\text { by connectional }\end{array}$ & $\begin{array}{l}\text { can be expressed } \\
\text { by heterogeneous }\end{array}$ \\
\hline
\end{tabular}

We present that it is equivalent to work with adjoint triples (in multi-adjoint approach) and to consider isotone and left-continuous functions (in our heterogeneous approach). Nevertheless, our environment allows us to use different complete lattice for every object, different complete lattice for every attribute and different poset for every table field.

The complexity of our approach depends on the number of different degrees for all objects and attributes. The heterogeneous formal concepts was provided by a brute-force approach. We have generated all possible functions and output those for which was fulfilled definition of heterogenenous formal concept. Anyway, Bělohlávek shows how to deal with the problem of generating all concepts of a fuzzy concept lattice in [4] with better complexity. A fast bottom-up algorithm to compute all concepts of a fuzzy closure operator is presented in [7]. We would like to modify and generalize these algorithms for our heterogeneous approach, too. And in this way we will make assumption of not linearly ordered set of truth degrees. Then it is fruitful to apply it on real-world data.

We would like to put emphasis that there is a similarly called approach working with multi-adjoint concept lattices based on heterogeneous conjunctors. This is done by Medina and Ojeda-Aciego in [22]. The difference is following. Multi-adjoint concept lattices work with different lattices too, but only for sets of attributes and objects. The objects and the attributes are evaluated in two different lattices and on heterogeneous conjunctors. Finally both lattices are embedded to the new so-called connected lattice. Thus the concept lattice utilizes the same lattice for objects and attributes.

The next interesting connection is clarifying the relationship of our heterogeneous approach to Bělohlávek \& Vychodil's fuzzification working with truth-stressers, so-called hedges proposed in [9] and [10]. In [20] it is shown that generalized concept lattices cover them in some sense but it seems that this new approach make this relationship more immediate.

The hedges is used in [16] as a tool to reduce the size of multi-adjoint concept lattices with heterogeneous conjunctors as unifying the approches introduced in [22] and [10].

Another relationship that seems to be interesting for future work is to consider heterogeneity in multi-adjoint concept multilattices that are more general structures as lattices [27]. The sets of multisuprema and multinfima are introduced and usefulness of such structures is noticed.

\section{Acknowledgement}

This work was partially supported by the grant VEGA 1/0832/12, by the Slovak Research and Development Agency under contract APVV-0035-10 "Algorithms, Automata, and Discrete Data Structures" by the Agency of the Slovak Ministry of Education for the Structural Funds of the EU, under project ITMS:26220120007. This work was also partially supported by the EUSFLAT Student Grants Program.

\section{References}

[1] L Antoni, S. Krajči, O. Krídlo, B. Macek, L. Pisková, Relationship between two FCA approaches on heterogeneous formal contexts. In Laszlo Szathmary, Uta Priss (Eds.), proceedings of the 9th International Conference on Concept Lattices and Their Applications (CLA 2012), Universidad de Malaga, pages 93-102, October 11-14, Malága (Spain), 2012. 
[2] L Antoni, S. Krajči, O. Krídlo, B. Macek, L. Pisková, On heterogeneous formal contexts, Fuzzy Sets and Systems (2013). In Press.

[3] R. Bělohlávek, Fuzzy concepts and conceptual structures: induced similarities. JCIS98, Vol. I, Durham, USA, pages 179-182, 1998.

[4] R. Bělohlávek, Algorithms for fuzzy concept lattices. In Proc. 4th Int. Conf. Recent Adv. Soft Comput., pages 200-205 Nottingham (U.K.), Dec. 12-13, 2002.

[5] R. Bělohlávek, Concept Lattices and Order in Fuzzy Logic, Annals of Pure and Applied Logic, 128: 277-298, 2004.

[6] R. Bělohlávek, Sup-t-norm and inf-residuum are one type of relational product: Unifying framework and consequences, Fuzzy Sets and Systems, 197: 45-58, 2012.

[7] R. Bělohlávek, B. De Baets, J. Outrata, V. Vychodil, Computing the Lattice of All Fixpoints of a Fuzzy Closure Operator, IEEE Transactions on Fuzzy Systems, 18 (3): 546-557, 2010.

[8] R. Bělohlávek, V. Sklenář, J. Zacpal, Crisply generated fuzzy concepts. In B. Ganter and R. Godin (Eds.), ICFCA 2005, Lecture Notes in Computer Science 3403, pages 268-283, Springer-Verlag, Berlin/Heidelberg, 2005.

[9] R. Bělohlávek, V. Vychodil, Reducing the size of fuzzy concept lattices by hedges. FUZZ-IEEE 2005, USA, pages 663-668, 2005.

[10] R. Bělohlávek, V. Vychodil, Formal concept analysis and linguistic hedges, International Journal of General Systems 41 (5): 503-532, 2012.

[11] S. Ben Yahia, A. Jaoua, Discovering knowledge from fuzzy concept lattice. In Kandel A., Last M., Bunke H., Data Mining and Computational Intelligence, Physica-Verlag, pages 169 190, 2001.

[12] A. Burusco, R. Fuentes-Gonzalez, The study of L-fuzzy concept lattice, Mathware 85 Soft Computing, 3: 209-218, 1994.

[13] M. E. Cornejo, J. Medina, E. Ramírez, A comparative study of adjoint triples, Fuzzy Sets and Systems, 211: 1-14, 2013.

[14] B.A. Davey, H.A. Priestley, Introduction to Lattices and Order, Cambridge University Press, 2002.

[15] B. Ganter, R. Wille, Formal Concept Analysis, Mathematical Foundation, Springer Verlag 1999.

[16] J. Konecny, J. Medina, M. Ojeda-Aciego, Using intensifying hedges to reduce size of multiadjoint concept lattices with heterogeneous conjunctors. In Laszlo Szathmary, Uta Priss (Eds.), CLA 2012, Universidad de Malaga, pages 245256, October 11-14, Malága (Spain), 2012.

[17] S. Krajči, Cluster based efficient generation of fuzzy concepts, Neural Network World, 13(5): 521-530, 2003.

[18] S. Krajči, A generalized concept lattice, Logic Journal of IGPL, 13 (5): 543-550, 2005.
[19] S. Krajči, The basic theorem on generalized concept lattice. In V. Snášel, R. Bělohlávek,eds., proceedings of the 2nd international workshop (CLA 2004), pages 25-33, Ostrava (Czech republic), 2004.

[20] S. Krajči, Every Concept Lattice With Hedges Is Isomorphic To Some Generalized Concept Lattice. In CLA 2005, Olomouc (Czech republic), pages 1-9, 2005.

[21] J. Medina, M. Ojeda-Aciego, Multi-adjoint tconcept lattices, Information Sciences, 180 (5): 712-725, 2010.

[22] J. Medina, M. Ojeda-Aciego: On multi-adjoint concept lattices based on heterogeneous conjunctors, Fuzzy Sets and Systems, 208: 95-110, 2012.

[23] J. Medina, M. Ojeda-Aciego, J. Ruiz-Calviño, Formal concept analysis via multi-adjoint concept lattices, Fuzzy Sets and Systems, 160(2): 130-144, 2009.

[24] J. Medina, M. Ojeda-Aciego, A. Valverde, P. Vojtáš, Towards biresiduated multi-adjoint logic programming, Lect. Notes in Artificial Intelligence 3040, pages 608-617, 2004.

[25] J. Medina, M. Ojeda-Aciego, P. Vojtáš, Multi-adjoint logic programming with continuous semantics. Logic programming and NonMonotonic Reasoning, $L P-N M R$ '01, Lect. Notes in Artificial Intelligence 2173, pages 351-364, 2001.

[26] J. Medina, M. Ojeda-Aciego, P. Vojtáš, Similarity-based unification: a multi-adjoint approach, Fuzzy Set and Systems, 146: 43-62, 2004.

[27] J. Medina, J. Ruiz-Calviño, Fuzzy formal concept analysis via multilattices: first prospects and results. In Laszlo Szathmary, Uta Priss (Eds.), CLA 2012, Universidad de Malaga, pages 69-79, October 11-14, Malága (Spain), 2012, (2012)

[28] J. Pócs, Note on generating fuzzy concept lattices via Galois connections, Information Sciences, 185 (1): 128-136, 2012.

[29] J. Pócs, On possible generalization of fuzzy concept lattices, Information Sciences, 210: 8998, 2012.

[30] S. Pollandt, Fuzzy Begriffe, Springer, 1997.

[31] S. Pollandt, Datenanalyse mit Fuzzy-Begriffen. In G. Stumme, R. Wille, Begriffliche Wissensverarbeitung. Methoden und Anwendungen, pages 72-98, Springer, Heidelberg, 2000.

[32] A. Popescu, A general approach to fuzzy concepts, Mathematical Logic Quarterly, 50 (3): 265-280, 2004.

[33] Z. Shmuely, The structure of Galois connections, Pacific Journal of Mathematics, 54 (2): 209-225, 1974. 